\title{
Replanteando el concepto de bioseguridad en Chile: bioseguridad versus bioprotección en el área biomédica
}

\author{
Isidora Mura-Jornet ${ }^{1}$, Zoltan von Bernath ${ }^{1}$, Jorge Tomás Schoffer 1,2*
}

La bioseguridad puede definirse como las prácticas, procedimientos y uso de equipo para asegurar condiciones adecuadas cuando se trabaja con microorganismos potencialmente infecciosos $u$ otros materiales biológicos de peligro (Public Health Agency of Canada, 2015). Pese a esta definición, el concepto ha sido objeto de polémica en el ámbito científico, puesto que no existe consenso entre las diferentes entidades y organizaciones $y$, de hecho, es utilizado en contextos tan distintos tales como la agricultura, la medicina, la biotecnología, el alimento y el terrorismo.

En países de habla hispana, como el caso de Chile, tanto las buenas prácticas dentro de un laboratorio como las medidas administrativas y reglamentarias relativas a la protección del mismo, recaen en el concepto "bioseguridad", mientras que en países de habla inglesa se identifican dos conceptos: biosafety y biosecurity (Aguilar-Elena et al., 2015). Por un lado, biosafety se define como la aplicación de una combinación de prácticas y procedimientos, instalaciones de laboratorio y equipo de seguridad cuando se trabaja con microorganismos potencialmente infecciosos, con el fin de proteger al personal de laboratorio y, a través de ellos, al público general. Por otro lado, biosecurity se define como la aplicación de una combinación de medidas que se abordan mediante la coordinación de procedimientos y prácticas administrativas, reglamentarias y de seguridad física aplicadas en un entorno de trabajo para reducir los riesgos de pérdida de material biológico, robo o mal uso causados por la mala gestión o mala rendición de cuentas y protección (World Health Organization, 2008). La poca claridad al diferenciar ambos conceptos no se limita solo a países de habla hispana, sino que tiene también un correlato al menos en la región del sudeste asiático, donde se reconoce la bioprotección como un "asunto crítico aun por reconocer en la región" (World Health Organization, 2008). Es posible que esta ambigüedad de definiciones exista también en otras regiones del planeta. Una propuesta para la traducción de biosecurity es "bioprotección" (Rivas, 2009; Aguilar-Elena et al., 2015), por lo que entenderemos biosafety como "bioseguridad". Bajo esta aclaración en las definiciones, es posible notar que la diferencia entre ellos está dada por la intencionalidad de la liberación del agente de riesgo biológico (ARB) (World Health Organization, 2006; Nordmann, 2010; World Health Organization, 2012).

Particularmente en Chile, el "Manual de Normas de Bioseguridad" desarrollado por CONICYT (Comisión Nacional de Investigación Científica y Tecnológica, 2008) es la principal referencia que trata respecto a temas de seguridad en los distintos laboratorios. En general, busca proponer mecanismos de protección frente a productos tóxicos, microorganismos patógenos, organismos vivos genéticamente modificados, material clínico contaminado con patógenos, materiales radioactivos, radiaciones no ionizantes, ultrasonido, vibraciones, ruidos y agentes químicos en general. Como vemos, se incluyen en un mismo documento agentes de riesgo biológicos (ARB), físicos y químicos. Por otra parte, se observa que no existe diferenciación entre bioseguridad y bioprotección y todo lo referido en el manual es asignado bajo el concepto de bioseguridad. El no tener una diferenciación clara de los conceptos instala un problema de base: es difícil pensar en la aplicación práctica de un concepto si este mismo no está definido ni diferenciado de otros similares o que puedan ser fuente de confusión.

Fecha de envío: 17 de Noviembre de 2016 - Fecha de aceptación: 16 de Diciembre de 2016

(1) Departamento de Ecosistemas y Medio Ambiente, Facultad de Agronomía e Ingeniería Forestal, Pontificia Universidad Católica de Chile, (2) Programa de Doctorado en Ciencias de la Agricultura, Dirección de Investigación y Posgrado, Facultad de Agronomía e Ingeniería Forestal, Pontificia Universidad Católica de Chile.

Autor de correspondencia: jtschoffer@uc.cl 
Los laboratorios del área de biomedicina representan riesgos de contraer enfermedades infecciosas para las personas que trabajan en estos. Existen casos conocidos de enfermedades contraídas en este tipo de instalaciones, como son el caso de la fiebre tifoidea, el cólera y el tétano, entre otras (World Health Organization, 2008). A lo anterior se puede sumar la liberación intencionada de un ARB. Es por esto que es necesario lograr una comprensión clara de los conceptos de bioseguridad y bioprotección. En esto, los actores del área biomédica deben ser los primeros responsables en establecer las estrategias necesarias para implementar de manera diferenciada protocolos de estos conceptos a nivel nacional. Lo anterior es por dos razones: (1) estos establecimientos son fuente de ARB, ya sea por liberación intencionada o accidental, y (2) porque desde un punto de vista clínico los médicos son los que tienen las competencias necesarias para establecer un rápido y acertado diagnóstico frente a posibles liberaciones intencionales de agentes biológicos que afecten la salud de las personas. Esto permitiría la ejecución de protocolos de respuestas adecuados, i.e., protocolos diferenciados para liberación accidental (bioseguridad) e intencional (bioprotección) de ARB.

\section{Referencias}

Aguilar-Elena R, González Sánchez J, Morchón R \& Martínez-Merino V. (2015). ¿Seguridad biológica o bioseguridad laboral? Gaceta Sanitaria 29, 473-473.
Comisión Nacional de Investigación Científica y Tecnológica. (2008). Manual de Normas de Bioseguridad 2a ed. Disponible en: http://www.conicyt.cl/fondecyt/files/2012/09/articles-30555_recurso_1.pdf

Nordmann BD. (2010). Issues in biosecurity and biosafety. International journal of antimicrobial agents 36 Suppl 1, S66-69.

Public Health Agency of Canada. (2015). Canadian Biosafety Standard. 2nd ed. Disponible en: http://canadianbiosafetystandards. collaboration.gc.ca/cbs-ncb/assets/pdf/cbsg-nldcb-eng.pdf

Rivas A. (2009). Biosafety y biosecurity. Punto y Coma 111, 2-4.

World Health Organization. (2006). Biorisk management: Laboratory biosecurity guidance. Disponible en: http://www.who.int/ csr/resources/publications/biosafety/WHO_CDS_EPR_2006_6.pdf

World Health Organization. (2008). Biosafety and Biosecurity in Health Laboratories. In Regional Workshop on Biosafety and Biosecurity in Health Laboratories, ed. World Health Organization. Pune, India.

World Health Organization. (2012). Laboratory Biorisk Management Strategic Framework for Action 2012-2016. Disponible en: http://apps.who.int/iris/bitstream/10665/70878/1/WHO_ HSE_2012.3_eng.pdf 